PROCEEDINGS OF THE

AMERICAN MATHEMATICAL SOCIETY

Volume 126, Number 6, June 1998, Pages 1821-1825

S $0002-9939(98) 04245-2$

\title{
RECURSIVE CONDITION FOR POSITIVITY OF THE ANGLE FOR MULTIVARIATE STATIONARY SEQUENCES
}

\author{
A. MAKAGON, A. G. MIAMEE, AND B. S. W. SCHRÖDER
}

(Communicated by Stanley Sawyer)

\begin{abstract}
In this note a recursive type condition for positivity of the angle between past and future for $q$-variate stationary sequences is provided. In the case $q=2$ it gives a simple different proof of a result due to Solev and Tserkhtsvadze on basicity of bivariate stationary sequences.
\end{abstract}

Let $Z$ denote the set of all integers, $C$ be the set of complex numbers and $C^{q}$ be the Cartesian product of $q$ copies of $C$. The elements of $C^{q}$ will be identified with column vectors. By $L^{2}\left(C^{q}\right)$ we will denote the Hilbert space of all $C^{q}$ valued functions on $(-\pi, \pi]$ that are square integrable w.r.t. the Lebesgue measure $d t$. Let $X=\left\{X^{k}(n) \in H: \quad n \in Z, \quad k=1, \ldots, q\right\}$ be a $q$-variate $(q<\infty)$ stationary sequence in a Hilbert space $H$; i.e. for every $k, j=1, \ldots, q$, the inner product $\left(X^{k}(n), X^{j}(m)\right)$ depends only on $n-m$. Let $M(X)=\overline{s p}\left\{X^{k}(n): k=1, \ldots, q, n \in\right.$ $Z\}, M_{+}(X)=\overline{s p}\left\{X^{k}(n): k=1, \ldots, q, n \geq 0\right\}$ and $M_{-}(X)=\overline{s p}\left\{X^{k}(n): k=\right.$ $1, \ldots, q, n<0\}$.

Definition 1. A stationary sequence $X$ is said to be of positive angle iff

$$
\sup \left\{|(x, y)|: x \in M_{+}(X), y \in M_{-}(X),\|x\|=\|y\|=1\right\}<1 .
$$

If a stationary sequence $X$ has a spectral density $F^{\prime}$ w.r.t. the Lebesgue measure (cf. [4]), then any $q \times q$ matrix $G$ satisfying $G(t)^{*} G(t)=F^{\prime}(t)$, dt-a.e., will be called a square root of $F^{\prime}$. Note that for every $x \in C^{q}, G(\cdot) x \in L^{2}\left(C^{q}\right)$. From [7], Theorem 4.5, it follows that $X$ is of positive angle iff there exists a constant $C$ such that for every $C^{q}$-valued trigonometric polynomial $f$

$$
\int_{-\pi}^{\pi}\left|G(t) P_{+} f(t)\right|^{2} d t \leq C \int_{-\pi}^{\pi}|G(t) f(t)|^{2} d t
$$

where $P_{+}$is the orthogonal projection in $L^{2}\left(C^{q}\right)$ onto $L_{+}^{2}\left(C^{q}\right)=\overline{s p}\left\{e^{i n \cdot} e_{k}: n \geq\right.$ $0, k=1, \ldots, q\}$.

Definition 2. The class of all $q \times q$ matrix valued functions $G$ with $G(\cdot) x \in L^{2}\left(C^{q}\right)$ for all $x \in C^{q}$, such that (2) holds true for some $C$ and all $C^{q}$-valued trigonometric polynomials $f$ will be denoted $\mathcal{P} A(q)$.

Received by the editors April 26, 1996 and, in revised form, December 4, 1996.

1991 Mathematics Subject Classification. Primary 60G12, 60G25.

Key words and phrases. Multivariate stationary sequence, prediction theory, positive angle.

This research was supported by ONR Grant No. N 00014 - 89 - J - 1824.

The second author was supported by Army Research Office grant DAAH 04-96-1-0027.

The third author was supported by ONR Grant No. N $00014-95-1-0660$.

(c) 1998 American Mathematical Society 
It is well known that if a $q$-variate stationary sequence $X$ is of positive angle then the spectral measure $F$ of $X$ is absolutely continuous w.r.t. the Lebesgue measure. Therefore $X$ is of positive angle iff there is a square root $G$ of the spectral density of $X$ such that $G \in \mathcal{P} A(q)$. If $q=1$ then in [2] it was proved that $X$ is of positive angle iff $F^{\prime}=|G|^{2}$ satisfies the so-called $A_{2}$ condition, i.e. there is a constant $C$ such that for each intrerval or its complement $I \subset(-\pi, \pi]$

$$
\left(\int_{I}|G(t)|^{2} d t\right)\left(\int_{I}|G(t)|^{-2} d t\right) \leq C|I|^{2}
$$

where $|I|$ is the length of $I$.

In 1986 Solev and Tserkhtsvadze [8] obtained necessary and sufficient conditions for positivity of the angle for full rank bivariate stationary sequences. The conditions were in terms of the coefficients of a triangular square root of $F^{\prime}$. The main idea of [8] can be summarized as follows.

Theorem 1 ([8]). Let $G=\left[\begin{array}{ll}\sigma & 0 \\ \tau & r\end{array}\right]$. The following conditions are equivalent:

1. $G$ belongs to $\mathcal{P} A(2)$.

2. $|\sigma|^{2}$ and $|r|^{2}$ satisfy the $A_{2}$ condition and the mapping

$$
\mathcal{A}: f \longrightarrow\left(\tau P_{+} \frac{1}{\sigma}-r P_{+} \frac{\tau}{\sigma r}\right) f
$$

extends to a continuous operator in $L^{2}(C)$.

3. $|\sigma|^{2}$ and $|r|^{2}$ satisfy the $A_{2}$ condition and the two mappings

$$
\begin{gathered}
\mathcal{A}_{-}: f \longrightarrow r P_{-} \frac{\tau}{r} P_{+} \frac{1}{\sigma} f, \\
\mathcal{A}_{+}: f \longrightarrow r P_{+} \frac{\tau}{r} P_{-} \frac{1}{\sigma} f
\end{gathered}
$$

extend to continuous operators in $L^{2}(C)$.

Having the above lemma the analytic conditions for the positivity of the angle provided by Solev and Tserkhtsvadze follow readily from Fefferman's theorem on the conjugate space of $H^{1}$ (see [1]).

Below we prove a version of the theorem above for the $q$-variate case. In particular this leads to a simpler proof of Theorem 1.

Recall that if $X$ is of positive angle then $X$ is so-called $J_{0}$ regular ([6]) and hence the range of the spectral density $F^{\prime}=\frac{d F}{d t}$ is constant $d t$-a.e. and for each $x$ in the range the function $\left|G^{\#}(\cdot) x\right|^{2}$ is integrable (see e.g. [3], Section 5), where $G^{\#}$ stands for the generalized inverse matrix. Therefore, without loss of generality we can assume that the sequence $X$ has a density which is of full rank.

Lemma 1. Suppose that $G^{-1}$ exists dt-a.e. Then $G \in \mathcal{P} A(q)$ if and only if the operator $G P_{+} G^{-1}$ sending $f(\cdot) \longrightarrow G(\cdot) P_{+} G^{-1}(\cdot) f(\cdot)$ is bounded in $L^{2}\left(C^{q}\right)$.

Proof. If $G \in \mathcal{P} A(q)$ then from the remark preceding the lemma it follows that $G^{-1}(\cdot) x \in L^{2}\left(C^{q}\right)$ for all $x \in C^{q}$. Moreover the inequality (2) extends to all $f$ such that $G(\cdot) f(\cdot) \in L^{2}\left(C^{q}\right)$. Letting in (2) $f=G^{-1} g$, where $g$ is a trigonometric polynomial, we obtain

$$
\int_{-\pi}^{\pi}\left|G(t)\left(P_{+} G^{-1}(t) g(t)\right)\right|^{2} d t \leq C \int_{-\pi}^{\pi}|g(t)|^{2} d t .
$$


So $G P_{+} G^{-1}$ is bounded. Conversely, if (4) extends to $L^{2}\left(C^{q}\right)$, then letting $g=G f$, where $f$ is a trigonometric polynomial we obtain (2).

Theorem 2. Suppose that $G(t)=\left[g_{i j}(t)\right]_{i, j=1, \ldots, q}$ is an invertible lower triangular matrix for every $t \in(-\pi, \pi]$. Denote $G^{-1}(t)=H(t)=\left[h_{i j}(t)\right]_{i, j=1, \ldots, q}$. Let $G_{u}(t)$ and $G_{l}(t)$ be the $(q-1) \times(q-1)$ matrices obtained from $G(t)$ by removing the $q$-th row and the $q$-th column, and the 1-st row and the 1-st column, respectively. Then $G \in \mathcal{P} A(q)$ if and only if

i) $G_{u} \in \mathcal{P} A(q-1)$,

ii) $G_{l} \in \mathcal{P} A(q-1)$,

iii) the mapping $f \longrightarrow \sum_{j=1}^{q} g_{q j} P_{+} h_{j 1} f$ extends to a continuous operator in $L^{2}(C)$.

Proof. Note that since we are working with lower triangular matrices $H_{u}=\left(G_{u}\right)^{-1}$ and $H_{l}=\left(G_{l}\right)^{-1}$. Write $G$ as a sum of two $q \times q$ matrices

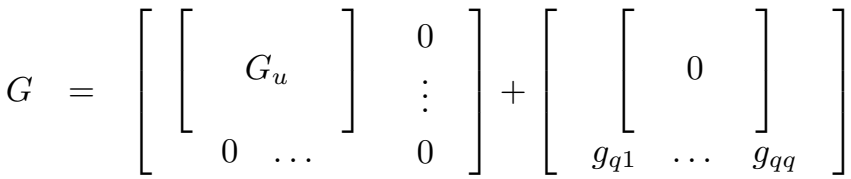

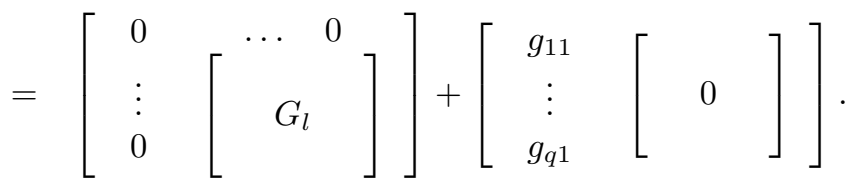

If we write $G^{-1}$ as

$$
G^{-1}=\left[\begin{array}{c}
h_{11} \\
\vdots \\
h_{q 1}
\end{array}\left[\begin{array}{l}
0 \\
\end{array}\right]+\left[\begin{array}{cc}
0 & \\
\vdots &
\end{array}\right]\right.
$$

then we obtain that

$$
\begin{aligned}
G P_{+} G^{-1}\left[\begin{array}{c}
f_{1} \\
f_{2} \\
\vdots \\
f_{q}
\end{array}\right] & =G P_{+} G^{-1}\left[\begin{array}{c}
f_{1} \\
0 \\
\vdots \\
0
\end{array}\right]+G P_{+} G^{-1}\left[\begin{array}{c}
0 \\
f_{2} \\
\vdots \\
f_{q}
\end{array}\right] \\
& =\left[\begin{array}{c}
G_{u} P_{+}\left[\begin{array}{c}
h_{11} f_{1} \\
\vdots \\
h_{q-1,1} f_{1}
\end{array}\right] \\
\sum_{j=1}^{q} g_{q j} P_{+} h_{j 1} f_{1}
\end{array}\right]+\left[\begin{array}{c}
f_{2} \\
G_{l} P_{+} G_{l}^{-1}\left[\begin{array}{c}
\vdots \\
f_{q}
\end{array}\right]
\end{array}\right] .
\end{aligned}
$$

Therefore $G P_{+} G^{-1}$ is bounded in $L^{2}\left(C^{q}\right)$ iff

A) $G_{l} P_{+} G_{l}^{-1}$ is bounded in $L^{2}\left(C^{q-1}\right)$,

B) $f \rightarrow G_{u} P_{+}\left[\begin{array}{c}h_{11} f \\ \vdots \\ h_{q-1,1} f\end{array}\right]$ is bounded from $L^{2}(C)$ to $L^{2}\left(C^{q-1}\right)$ and

C) $f \rightarrow \sum_{j=1}^{q} g_{q j} P_{+} h_{j 1} f$ is bounded in $L^{2}(C)$. 
If now we write

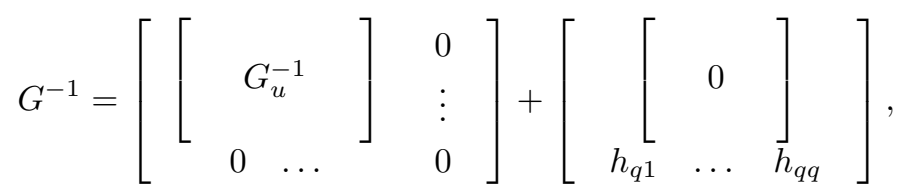

then

$$
\begin{aligned}
G P_{+} G^{-1}\left[\begin{array}{c}
f_{1} \\
\vdots \\
f_{q}
\end{array}\right]= & {\left[G_{u} P_{+} G_{u}^{-1}\left[\begin{array}{c}
f_{1} \\
\vdots \\
f_{q-1}
\end{array}\right]\right] } \\
0 & {\left[\begin{array}{c}
0 \\
\vdots \\
0 \\
\sum_{j=1}^{q} g_{q j} P_{+} \phi_{j}
\end{array}\right]+\left[\begin{array}{c}
0 \\
\vdots \\
0 \\
g_{q q} P_{+} \sum_{j=1}^{q} h_{q j} f_{j}
\end{array}\right] }
\end{aligned}
$$

where $\phi_{j}$ is the $j$-th coordinate of $G_{u}^{-1}\left[\begin{array}{c}f_{1} \\ \vdots \\ f_{q-1}\end{array}\right]$. This shows that if $G P_{+} G^{-1}$ is bounded in $L^{2}\left(C^{q}\right)$ then $G_{u} P_{+} G_{u}^{-1}$ is bounded in $L^{2}\left(C^{q-1}\right)$, which combined with A) and C) and Lemma 1 proves the necessity of conditions i) - iii).

Conversely, suppose that the conditions i) - iii) hold. Since $G_{u} P_{+} G_{u}^{-1}$ is bounded, its restriction

$$
G_{u} P_{+} G_{u}^{-1}\left[\begin{array}{c}
f \\
0 \\
\vdots \\
0
\end{array}\right]=G_{u} P_{+}\left[\begin{array}{c}
h_{11} f \\
\vdots \\
h_{q-1,1} f
\end{array}\right]
$$

is also bounded and we conclude that the conditions A), B) and C) are satisfied. Thus $G P_{+} G^{-1}$ is bounded, which in view of Lemma 1 completes the proof.

Solev and Tserkhtsvadze's Theorem 1 is a simple consequence of the theorem above. It is enough to note that under the assumptions of Theorem 1

$$
G^{-1}=\left[\begin{array}{cc}
\frac{1}{\sigma} & 0 \\
-\frac{\tau}{r \sigma} & \frac{1}{r}
\end{array}\right]
$$

For the equivalence of the second and third conditions observe first that if $\sigma, r \in$ $\mathcal{P} A(1)$ then $r P_{-} \frac{1}{r}=1-r P_{+} \frac{1}{r}$ and similarly $\sigma P_{-} \frac{1}{\sigma}$ is bounded and so $\mathcal{A}_{-}=r P_{-} \frac{1}{r} \mathcal{A}$ and $\mathcal{A}_{+}=-\sigma P_{-} \frac{1}{\sigma} \mathcal{A}$ are bounded. Conversely, boundedness of $\mathcal{A}_{+}$and $\mathcal{A}_{-}$clearly implies the boundedness of $\mathcal{A}$ for $\mathcal{A}=\mathcal{A}_{-}-\mathcal{A}_{+}$.

\section{REFERENCES}

1. Fefferman, C. (1971). Characterizations of bounded mean oscillation. Bull. Amer. Math. Soc. 77, 587-588. MR 43:6713

2. Hunt, R. A., Muckenhoupt, B. and Wheeden, R. L. (1973), Weighted norm inequalities for the conjugate function and Hilbert transform, Trans. Amer. Math Soc. 176, 227-251. MR 47:701

3. Makagon, A. and Salehi, H. (1989), Notes on infinite dimensional stationary sequences. Probability Theory on Vector Spaces IV, Lecture Notes in Math. 1391, Springer-Verlag, 200-238. MR 91i:60103 
4. Masani, P. and Wiener, N. (1957-58), The prediction theory of multivariate stochastic processes I and II, Acta Math. 98, 111 - 150, and 99, 93 - 137. MR 20:4323; MR 20:4325

5. Miamee, A. G. (1986), On the Angle between Past and Future for Multivariate Stationary Stochastic Processes, J. Mult. Anal. 20, 205 - 219. MR 88f:60074

6. Miamee, A. G. and Pourahmadi, M. (1987), Degenerate multivariate stationary processes: Basicity, Past and Future and Autoregressive Representation, Sankhya Ser A. 49, 316-334. MR 91b:62185

7. Pousson, H. R. (1968). Systems of Toeplitz operators on $H^{2}$, II, Trans. Amer. Math. Soc. 133, 527 - 536. MR 37:3377

8. Solev, V. N. and Tserkhtsvadze, K. A. (1986), A condition for a stationary vector sequence to be a basis (Russian), Zap. Nauchn. Sem. Leningrad. Otdel. Mat. Inst. Steklov. (LOMI) 153. MR 88b:60096

Department of Mathematics, Hampton University, Hampton, Virginia 26668

E-mail address: makagon@huajai.cs.hamptonu.edu

E-mail address: miamee@cs.hamptonu.edu

Current address, B. Schröder: Program of Mathematics and Statistics, Louisiana Technical University, Ruston, Louisiana 71272

E-mail address: Schroder@engr.LaTech.edu 